\title{
Estimation of Human Hip and Knee Multi-Joint Dynamics Using the LOPES Gait Trainer
}

\author{
Bram Koopman, Edwin H. F. van Asseldonk, Member, IEEE, and Herman van der Kooij, Member, IEEE
}

\begin{abstract}
In this study, we present and evaluate a novel method to estimate multi-joint leg impedance, using a robotic gait training device. The method is based on multi-input-multi-output system identification techniques and is designed for continuous torque perturbations at the hip and knee joint simultaneously. Eight elderly subjects (age 67-82) performed relax and position tasks in three different leg orientations. Multi-joint impedance was estimated nonparametrically and was subsequently modeled in terms of inertia and (inter)joint stiffness and damping. The results indicate that all stiffness and damping parameters were significantly higher during the position task compared to the relax task. The majority of the stiffness and damping parameters were not significantly affected by leg orientation. The results also emphasize the importance of considering the visco-elastic coupling between joints when modeling multi-joint dynamics. Measuring joint stiffness with the same device that is used for robotic gait training allows convenient testing of joint properties in conjunction with the robotic gait training protocol. These measures might serve as a good basis for quantitative assessment and follow up of patients with abnormal joint stiffness due to neurological disorders, and may reveal how changes in these joint properties affect their gait function.
\end{abstract}

Index Terms-Joint impedance, joint stiffness, multiinput-multi-output (MIMO) system identification, multi-joint impedance, robotic gait trainer.

\section{INTRODUCTION}

$\mathbf{O}$ UR ability to resist perturbations during postural control, or during coordinated movements like walking, greatly depends on our mechanical joint properties. Joint properties can be characterized by their mechanical impedance, which (in biomechanics) is often defined as the dynamic behavior between joint torque and angular displacement [1]. Experiments to quantify joint impedance typically involve mechanically perturbing

Manuscript received July 30, 2015; revised January 30, 2016; accepted April 12, 2016. Date of publication August 18, 2016; date of current version August 18, 2016. This paper was recommended for publication by Editor A. Kheddar and Guest Editors D. Kulic and G. Venture upon evaluation of the reviewers' comments. This study was supported in part by the Dutch Ministry of Economic Affairs and Province of Overijssel, The Netherlands, under Grant PID082004 and by the EU within the EVRYON Collaborative Project (Evolving Morphologies for Human-Robot Symbiotic Interaction) under Project FP7-ICT2007-3-231451.

B. Koopman was with University of Twente, Enschede 7500AE, The Netherlands (e-mail: bramkoopman2@gmail.com).

E. H. F. van Asseldonk is with the Department of Biomechanical Engineering, University of Twente, Enschede 7500AE, The Netherlands. (e-mail: e.h.f.vanasseldonk@utwente.nl).

H. van der Kooij is with the Department of Biomechanical Engineering, University of Twente, Enschede 7500AE, The Netherlands, and also with the Department of Biomechanical Engineering, Delft University of Technology, Delft 2628 CD, The Netherlands (e-mail: h.vanderkooij@utwente.nl).

This paper has supplementary downloadable material available at http://ieeexplore.ieee.org. It summarizes the model that is used to describe the dynamics of the human that is connected to the LOPES.

Digital Object Identifier 10.1109/TRO.2016.2572695 the joint in a controlled manner, measuring the motions and torques, and applying system identification techniques. These experiments have been performed on a wide variety of joints, including the ankle, wrist, elbow, and knee, and revealed that the joint impedance could be described by inertial, viscous, and elastic properties (for small displacements) [2].

Often, a distinction is made between the impedance measured in a passive joint (passive impedance) and in a joint with a certain level of muscle contraction (active impedance). The passive component is ascribed to the inertia of moving segments and the dynamic properties of anatomical structures like joint capsules, ligaments, connective tissues, and inactive muscles. The active impedance is caused by the properties of activated muscle groups acting around the joint. Others try to decompose the impedance into an intrinsic and reflexive part. Here the intrinsic part arises from the mechanical properties of passive tissues and active muscles, whereas the reflexive part arises from reflexes, which lead to changes in muscle activation levels and consequently contribute to joint impedance.

Joint perturbation experiments on nondisabled subjects showed that the joint impedance depends on several factors, such as muscle contraction levels [3]-[5], joint angle [6], [7], movement amplitude [5], [8], [9] and perturbation bandwidth [10], [11]. These dependences can be explained by several underlying physiological mechanisms. For example, the angular dependency is likely to be related to the nonlinear behavior of passive structures [12], the change in the overlap between the myosin and actin filaments [13], and changes in the muscle moment arm [14]. The increase with contraction level is ascribed to the summation of the stiffness of parallel arranged cross- bridges [15]. Cross-bridges are also thought to cause a high stiffness during low amplitude perturbations, due to elastic stretch during the initial stages of the stretch, and a lower stiffness during large amplitude perturbations, due to detaching and reconnecting muscle filaments [16]. The bandwidth dependency is related to our capability to modulate our reflexive activity. For low bandwidth perturbations, humans increase their spinal reflexes (muscle spindles and Golgi tendon organs) and effectively increase their joint impedance. At higher frequencies this is limited due to instabilities arising from reflexive time delays [17]. These reflexes also appear to have larger gains for small amplitude perturbations, compared to larger perturbations [18].

A thorough understanding of joint stiffness and its variation with posture, muscle activation levels, environmental circumstance, or diseases might prove useful for several applications. Knowledge about the way we modulate our joint impedance during different functional tasks such as locomotion, running, or balance control can benefit the design of activated prostheses or orthoses [19] and can be used to improve the control of 
paralyzed limbs using functional electrical stimulation [20]. Additionally, joint impedance measurements can serve as a good basis for quantitative assessment and followup of patients with ligament injuries [21] or abnormal joint stiffness (spasticity) due to neuromuscular disorders like spinal cord injury (SCI), stroke, cerebral palsy, or Parkinson's disease [22], [23]. Quantitative impedance measures were also demonstrated to have an intrasubject reliability that was as good as, or better than, most clinical measures [24]. This makes them also suitable as an objective indicator of the effectiveness of different types of rehabilitational interventions.

As mentioned above, several factors influence joint impedance. Still, the main factor is task instruction [25]. Task instruction is inherently linked to the applied perturbation type; force tasks are used during position perturbations and position tasks during force perturbations [26]. So far, most studies use position perturbations in combination with a torque/force task. These studies often focus on the effect of muscle contraction levels on joint stiffness. We believe that torque/force perturbations present a more natural disturbance, since the effort of the subjects is reflected in their performance, whereas during a position perturbation the subjects have no influence on the performed movement. Therefore, in this study, we will use torque perturbations in combination with relax and position tasks to determine the range of joint impedance.

Conventional methods for measuring joint impedance typically have been applied to a single joint. However, due to the presence of bi-articular muscles and heteronymous reflexes [27], the joint impedance of one joint is also influenced by the angular position or movement of the adjacent joint. Several studies have illustrated the effect of bi-articular muscles by the change in the hip-torque versus hip-angle curve, for different orientations of the knee joint and vice versa [28], [29]. Such relations are often used to describe how these elastic elements can serve as an energy storage and release mechanism, rather than quantifying the joint mechanics in terms of stiffness and damping [30]. Also, these studies are only performed for passive movements in which the joints are slowly moved through their range of motion, rather than applying perturbations. For the upper extremities this multi-joint characteristic of joint impedance has been acknowledged, and multi-input-multioutput (MIMO) techniques have been used for the estimation of multi-joint stiffness (or "endpoint stiffness") [31], [32].

The goal of this study is to use similar MIMO techniques to develop and evaluate an approach to assess multi-joint leg impedance using a robotic gait training device. The hip and knee joint will be mechanically perturbed simultaneously, and the effect on both hip and knee angular displacement will be measured. Subsequent MIMO system identification techniques in the frequency domain will be used to distinguish between single-joint and multi-joint effects. A significant coupling between both joints is expected due to the mechanical connection and the presence of bi-articular muscles and heteronymous reflexes. We use closed-loop identification techniques, which are appropriate when continuous torque disturbances are used. We also investigate the effect of task instruction (relax versus positions task) and leg posture on hip and knee stiffness.
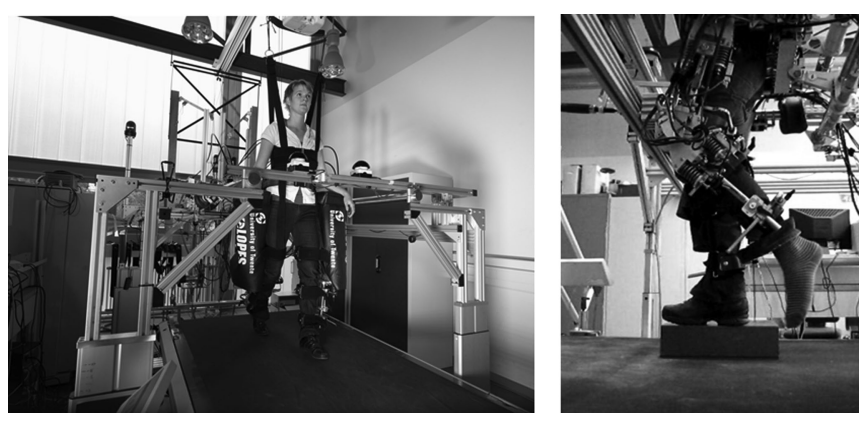

Fig. 1. Left: LOPES robotic gait trainer. It comprises a bilateral exoskeleton, which is force controlled using "series elastic actuation" via Bowden cables, and is capable of applying torque perturbations to the hip and knee joint. Right: Experimental setup. During the perturbation experiments the subjects stood with the nonperturbed leg on a box such that the perturbed leg could move freely.

\section{METHODS}

\section{A. Subjects}

Eight elderly subjects between the age of 67 and 82 [age $74.8 \pm 5.3$ (mean \pm std), seven males, one female, weight 81.9 $\pm 11.6 \mathrm{~kg}]$ participated in this study. None of the subjects had symptoms of neurological or orthopedic dysfunction and all gave informed consent before participating in the experiments. The protocol was in accordance with the Declaration of Helsinki.

\section{B. Apparatus}

To apply hip and knee perturbations, the LOPES (Lower Extremity Powered Exoskeleton) was used. The LOPES (see Fig. 1) is an exoskeleton type robotic gait trainer with eight actuated degrees of freedom (DoF) and was initially designed to provide supported treadmill training for stroke patients. It is force controlled using "series elastic actuation" (SEA) via Bowden cables with the elastic element on the joint (thus after the Bowden cable). The applied joint torques are calculated from the deflection of the springs of the SEA, eliminating the need to make an assumption regarding, and compensate for, the friction in the cables [33]. Although the LOPES is designed to have a transparent (backdriveable) mode in which no torques are applied when the patient requires no assistance, this mode will not be used since in this study the LOPES is used to apply torque perturbations.

MATLAB xPC (Mathworks, Natick, MA, USA) is used to control the applied torques by the exoskeleton joints at 1000 Hz. Potentiometers fitted to the LOPES joints record the joint angles. In this experiment perturbations will be applied to the knee and hip joint. The angles and exerted joint torques were sampled at $100 \mathrm{~Hz}$ and stored for later processing. Horizontal and vertical pelvic movements were mechanically constrained, so movements in these DoF were not possible. A bias torque was used to keep the hip ab/adduction in a neutral position.

\section{Electromyography}

Muscle activity was recorded from six muscles acting about the knee and hip joint (Porti 16-5, supplier: TMS International, Enschede, The Netherlands). We measured EMG levels of the mono-articular muscles: 1) vastus lateralis and 
2) gluteus maximus and biarticular muscles: 3) rectus femoris, 4) semitendinosis, 5) biceps femoris and 6) gastrocnemius. Seniam guidelines [34] were followed for skin preparation and placement of the disc-shaped solid-gel $\mathrm{Ag} / \mathrm{AgCl}$ electrodes (in a bipolar configuration). The EMG signals were sampled at $1024 \mathrm{~Hz}$, and digitally stored for further processing. A sync signal was used to synchronize the EMG and LOPES data. All recordings were performed on the left leg only. As a reference for the EMG levels, maximum voluntary contraction (MVC) levels were collected according to the Seniam guidelines [34]. Some of the cuffs of the LOPES were placed over the electrodes. To account for any changes in EMG levels due to the compression of the skin under the cuffs, the MVC levels were recorded in the LOPES. The obtained MVC levels were used to scale the EMG levels during the different conditions.

\section{Experimental Protocol}

Before positioning the subject in the LOPES, different anthropometric measurements were taken to adjust the exoskeleton segments lengths. Additionally, the position of the cuffs was adjusted in two DoFs to align the subject's hip and knee axis with the exoskeleton joints. Next, the subject was positioned into the LOPES and his trunk, thigh, and upper- and lower-shank were strapped to the exoskeleton. To let the subject become familiar with the device, every subject was allowed $5 \mathrm{~min}$ to freely move and walk in the device while it was operated in the zero-impedance mode [35]. This period was also used to correct for any small misalignment between the human and exoskeleton joints. During the perturbation experiments, the subject stood with his nonperturbed leg on a box, while holding onto two parallel bars for support (see Fig. 1). The height of the box was $15 \mathrm{~cm}$, which was sufficient to clear the foot of the perturbed leg.

1) Experimental Conditions: Subjects were asked to perform two different tasks: a relax task and a position task. While performing each task, the LOPES applied continuous torque perturbations to the hip and knee. During the position task, the hip and knee angles were displayed on a screen in real time and the subject was instructed to keep the deviations as small as possible. During the relax task, the subject was asked not to interfere with the applied perturbations and the screen was turned off to prevent any distractions. To study the effect of leg orientation on the joint impedance, the position and relax tasks were performed at three different leg orientations [hip/knee angles of $5 /-55^{\circ}, 25 /-35^{\circ}$, and $25 /-15^{\circ}$; see Fig. 2(c) and (d) for the definitions of the angles]. These three leg configurations represent the leg orientation during natural gait: 1) just after toe off, 2) during swing, and 3) prior to heel strike. For each combination of task and configuration (relax versus position, at three positions, thus six conditions), we applied two perturbations (see the description of "perturbation signal"). For the relax task these perturbations were applied in one trial each. For the position task each perturbation was split into two trials to prevent fatigue. All six conditions were randomized, with a resting period of at least $2 \mathrm{~min}$ in between conditions. Since the active impedance does not differ between dominant and nondominant

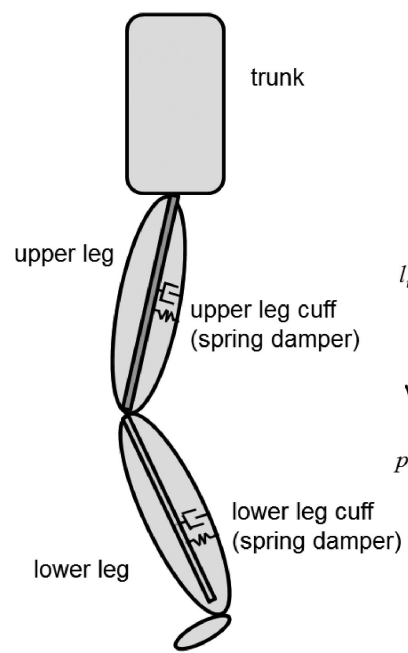

(a)

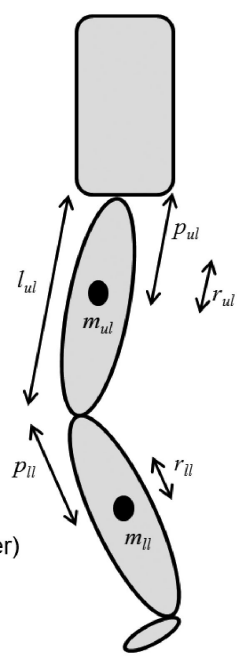

(b)

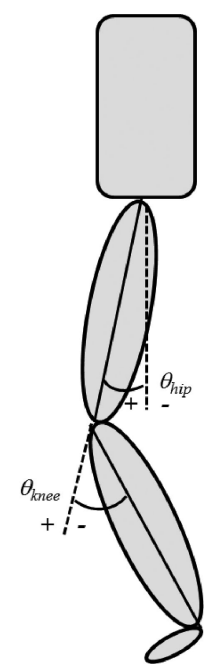

(c)

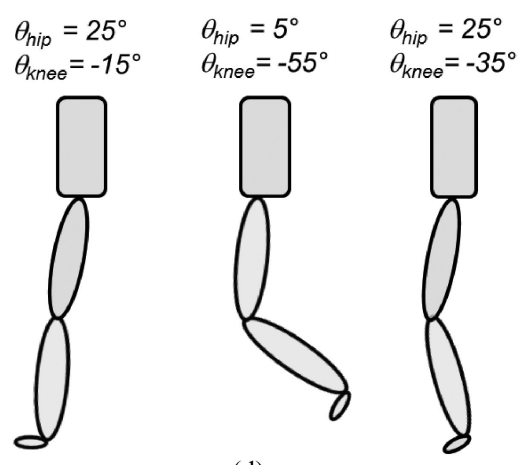

(d)

Fig. 2. Model parameters and tested leg orientations. (a) Schematic representation of the dynamic model that is used for parameter estimation. It represents the human leg that is attached to the LOPES. Both the LOPES and the human are modeled as a double pendulum. The pendulum of the human and the exoskeleton are connected by means of parallel spring-damper combinations at the thigh and shank, which represent the dynamic behavior of the cuffs. (b) Fixed model parameters of the double pendulum that represents the human leg. (c) Definitions of the hip $\left(\theta_{\text {hip }}\right)$ and knee angle $\left(\theta_{\text {knee }}\right)$. (d) Tested leg orientations, "end swing," "initial swing," and "mid swing."

limbs in healthy subjects [36], all perturbations were applied to the left leg.

During all conditions a bias torque was superimposed on the multisine (see the description of "perturbation signal"). The bias torque was used to compensate for the gravity of the leg and the exoskeleton. This allowed the subject to passively keep the hip and knee joint in the desired testing angle and prevented unwanted muscle contractions (especially during the relax task). The bias torques were set for every subject and condition individually.

As mentioned above, the SEA eliminates the need to make an assumption regarding, and compensate for, the friction in the Bowden cables. However, the joint of the exoskeleton itself has friction and the linkages have a certain mass and inertia. To obtain these dynamic properties of the exoskeleton, which are required for the estimation of the human dynamical properties [see supplementary material, (8)], a similar set of experiments was performed without a subject in the LOPES. In other words, 
we did not model the exoskeleton but used the estimated frequency response function (FRF) of the experiment without a subject in the LOPES to compensate for the dynamics of the exoskeleton. By doing so we eliminate any errors that might have occurred when we would have modeled the behavior of the exoskeleton.

2) Perturbation Signal: Quasi-random torque perturbations were used to prevent anticipatory muscle contractions. The perturbation signal was composed of multiple sinusoids. This multisine signal had a duration of $20 \mathrm{~s}$ and contained an equal amount of power at 35 specified frequencies $(0.05-10 \mathrm{~Hz}$, logarithmically spaced). The signal was generated offline and allowed for a well-defined frequency domain analysis. Because linear system identification techniques were used, large movement amplitudes should be avoided. Therefore the phases of the different sines of the perturbation signal were optimized by crest optimization [37]. To enable a comparison of the different conditions, and to justify a linear modeling approach, the amplitude of the multisine torque disturbance was adjusted for every subject and condition so that that the peak-to-peak amplitude of the resulting hip or knee angle did not exceed $15^{\circ}$. These adjustments were made prior to the actual experiments, by slowly ramping up the amplitude of the disturbance signal until the peak-to-peak amplitude of the knee or hip angle reached approximately $15^{\circ}$.

A general requirement to identify a MIMO system is that for each degree of freedom an independent perturbation is required. This implies that the system needs to be perturbed in two different manners. For the first perturbation, the perturbation signals on the hip and knee had the opposite sign, whereas for the second perturbation the perturbation signals had equal signs. For the relax task one trial was performed for each perturbation, consisting of seven repetitions (cycles) of the 20-s multisine signal. For the position task two trials were performed for each perturbation, consisting of four repetitions (cycles) of the multisine signal each (to prevent fatigue). The first cycle of each trial is discarded, to eliminate possible transients at the start of each trial, resulting in six repetitions per trial.

\section{E. Data Processing}

All signal processing was done with custom-written software in MATLAB (Mathworks, Natick, MA, USA).

1) Muscle Contraction Levels: The raw EMG recordings were bandpass filtered $(10-400 \mathrm{~Hz})$ with a second-order zerolag Butterworth filter to remove movement artifacts, full-wave rectified, and low-pass filtered with a second-order zero-lag Butterworth filter $(5 \mathrm{~Hz})$ to smooth the signal. The EMG was resampled to $100 \mathrm{~Hz}$ and synchronized to the LOPES data. Mean EMG levels were calculated for all the cycles of the perturbation signal. Next, these mean EMG levels were averaged for every condition to provide a single measure of muscle activity level per condition. Also, the standard deviation of the mean EMG levels per condition was calculated to provide a measure of the variability in EMG levels over the six different cycles.

2) Nonparametric MIMO System Identification, FRFs: The data from the six perturbation cycles were Fourier transformed and averaged over the six cycles to reduce the effects of noise.
Note that only the Fourier coefficients at the frequencies of the multisine that contained power were used for further processing. Next, MIMO frequency analysis techniques were used to obtain the nonparametric identification of the overall admittance. Although the admittance and its inverse, the impedance, technically refers to the relationship between force and velocity, in many studies on joint properties these terms refer to the relationship between force and position [1]. Here the admittance is defined as the causal dynamic relationship with torque as input and angle as output.

The overall admittance $H(s)$ of the system is defined according to

$$
\Theta(s)=T(s) \cdot H(s)
$$

where $\Theta(s)$ and $T(s)$ denote the Fourier transforms of the exoskeleton joint angles and applied joint torques, respectively, and $s$ is the Laplace variable. For the sake of simplicity, $(s)$ is omitted from this point forward. Since the total system (human+LOPES) represents a multivariate system with two inputs (torques) and two outputs (angles), the admittance FRF consists of a 2-by-2 matrix with FRFs. The FRFs are calculated according to

$$
\begin{aligned}
H & =T^{-1} \cdot \Theta \\
\widehat{H}_{\text {tot }} & =\left[\begin{array}{ll}
H \tau_{\text {hip }} \rightarrow \theta_{\text {hip }} & H \tau_{\text {hip }} \rightarrow \theta_{\text {knee }} \\
H \tau_{\text {knee }} \rightarrow \theta_{\text {hip }} & H \tau_{\text {knee }} \rightarrow \theta_{\text {knee }}
\end{array}\right] \\
& =\left[\begin{array}{ll}
\widehat{T}_{\text {hip }_{1}} & \widehat{T}_{\text {knee }_{1}} \\
\widehat{T}_{\text {hip }_{2}} & \widehat{T}_{\text {knee }_{2}}
\end{array}\right]^{-1} \cdot\left[\begin{array}{ll}
\widehat{\Theta}_{\text {hip }_{1}} & \widehat{\Theta}_{\text {knee }_{1}} \\
\widehat{\Theta}_{\text {hip }_{2}} & \widehat{\Theta}_{\text {knee }_{2}}
\end{array}\right]
\end{aligned}
$$

where $T_{\text {hip }}$ and $T_{\text {knee }}$ represent the Fourier transforms of the hip and knee torque. $\Theta_{\text {hip }}$ and $\Theta_{\text {knee }}$ represent the Fourier transforms of the exoskeleton joint angles. Superscript ${ }^{-1}$ denotes the inverse, subscript ${ }_{1}$ and ${ }_{2}$ indicate the Fourier transforms obtained during the first and second perturbation, and the circumflex ${ }^{\wedge}$ denotes an estimate. $H_{\tau_{\text {hip }} \rightarrow \theta_{\text {knee }}}$ denotes the calculated FRF between the applied hip torque and knee angle. The same perturbation torque signal was used for the hip and knee. Note that matrix $T$ must be invertible. Therefore, for the first perturbation, the perturbation signals on the hip and knee had opposite signs.

3) Noise-to-Signal Ratio (NSR): To determine whether it is justified to use time-invariant system-identification methods, we calculated the NSR in the frequency domain [38]. The NSR is the ratio of the remnant and the periodic response. Remnant can result from time-variant behavior and/or noise. Consequently, a small NSR indicates a consistent response of the system to the applied perturbation over the different cycles. It also indicates that the system is appropriately perturbed. The NSR is defined according to

$$
\mathrm{NSR}_{\text {hip }}=\frac{\sigma^{2} \Theta_{\text {hip }}}{\left|\Theta_{\text {hip }}\right|^{2}}
$$

where $\sigma^{2} \Theta_{\text {hip }}$ represents the variance of the remnant (i.e., the variance of the Fourier transforms of the exoskeleton hip angle over the different cycles) and $\Theta_{\text {hip }}$ represents the periodic 
response (i.e., the mean of the Fourier transforms of the exoskeleton hip angle over the different cycles). The NSR of the knee angular response is calculated in a similar way. The NSR is only calculated for the excited frequencies and averaged over the frequencies.

4) Cycle to Cycle Variability: Another measure that reflects the consistency of the response was provided by the standard deviation of the hip and knee angular response. The standard deviation was calculated across the six cycles for each perturbation. Next, the standard deviation values were averaged across the recorded time instances (2000 samples per $20 \mathrm{~s}$ ) to obtain one representative measure of the intercycle variability in the time domain.

5) Model Fit: To estimate the hip and knee impedance in terms of physiologically relevant parameters, a linear model was constructed. In this model the mechanical behavior of the complete system is represented by two double pendulums, one representing the exoskeleton's leg (LOPES pendulum) and one representing the subject's leg (human pendulum) [see Fig. 2(A)]. Both double pendulums are connected by means of parallel spring-damper combinations (Kelvin bodies), which represent the cuffs of the LOPES and the soft tissue of the legs. Each segment of the human pendulum has a mass (located at a certain distance from the joint) and a radius of gyration [see Fig. 2(b)]. We lumped the mono-articular and bi-articular effects of the muscles and connective tissue crossing the hip and knee joint into three pairs of parameters. The first pair describes the lumped contribution to rotational stiffness and damping of the knee $\left(K_{k}, B_{k}\right)$, the second pair describes the contribution to rotational stiffness and damping of the hip $\left(K_{h}, B_{h}\right)$, and the third pair describes the contribution to the symmetric visco-elastic coupling between the hip and knee (i.e., how does a change in knee angle influence the hip torque and vice versa) $\left(K_{c}, B_{c}\right)$. The definitions of the model parameters are listed in Table I. See the supplementary material for a detailed description of the model and the parameterized impedance transfer function (TF) of the human pendulum model $\left(H_{\text {model }}\right)$. The best model fit was obtained by minimizing the following criterion function:

$$
\text { model error }=\sum_{f}\left(\frac{\left|\log \left(\widehat{H}_{\text {human }}(f)\right)-\log \left(H_{\text {model }}(f)\right)\right|}{\left|\log \left(\widehat{H}_{\text {human }}(f)\right)\right|}\right)
$$

where $H_{\text {model }}$ represents the TF of the human pendulum model. $\hat{H}_{\text {human }}$ represents the impedance FRF of the human leg and is calculated from the total admittance $\left(\hat{H}_{\text {tot }}\right)$; see the supplementary material. The criterion function is only evaluated for the excited frequencies. The optimization was performed with an unconstrained nonlinear optimization routine and on all 4 FRFs that comprise $\hat{H}_{\text {human }}$ simultaneously. Since the NSR was relatively high at the frequencies above $3 \mathrm{~Hz}$, it was decided to only include all frequencies up to $3 \mathrm{~Hz}$ ( 25 frequencies in total).

To reduce the amount of model parameters that require fitting, the position of the center of mass, the radius of gyration, and the masses of the lower and upper leg were taken from empirical relations reported in the literature [39]. To account for some subject variability in physique, we introduced a scaling factor to
TABLE I

MODEL PARAMETERS

\begin{tabular}{|c|c|c|c|}
\hline & Description & Unit & Parameterization \\
\hline$K_{h}$ & Hip stiffness & $\mathrm{Nm} / \mathrm{rad}$ & Optimized \\
\hline$K_{k}$ & Knee stiffness & $\mathrm{Nm} / \mathrm{rad}$ & Optimized \\
\hline$K_{c}$ & $\begin{array}{l}\text { Multi-joint stiffness due to } \\
\text { bi-articular effect }\end{array}$ & $\mathrm{Nm} / \mathrm{rad}$ & Optimized \\
\hline$B_{h}$ & Hip damping & Nms/rad & Optimized \\
\hline$B_{k}$ & Knee damping & $\mathrm{Nms} / \mathrm{rad}$ & Optimized \\
\hline$B_{c}$ & $\begin{array}{l}\text { Multi-joint damping due to } \\
\text { bi-articular effect }\end{array}$ & $\mathrm{Nms} / \mathrm{rad}$ & Optimized \\
\hline$c$ & Scaling factor for $m_{\mathrm{u} 1}$ and $m_{11}$ & dimensionless & Optimized \\
\hline$l_{\mathrm{u} 1}$ & Length of the upper leg & $\mathrm{m}$ & Fixed (True value) \\
\hline$m_{\mathrm{ul}}$ & Mass of the upper leg & $\mathrm{kg}$ & $\begin{array}{l}\text { Fixed }(0.115 \cdot \text { body } \\
\text { mass })^{4}\end{array}$ \\
\hline$p_{\mathrm{u} 1}$ & $\begin{array}{l}\text { Position of the center of mass of } \\
\text { the upper leg }{ }^{2}\end{array}$ & $\mathrm{~m}$ & $\begin{array}{l}\text { Fixed }(0.425 \cdot \text { upper leg } \\
\text { length })^{4}\end{array}$ \\
\hline$r_{\mathrm{ul}}$ & $\begin{array}{l}\text { Radius of gyration of the upper } \\
\text { leg }\end{array}$ & $\mathrm{m}$ & $\begin{array}{l}\text { Fixed }(0.29 \cdot \text { upper leg } \\
\text { length })^{4}\end{array}$ \\
\hline$m_{11}$ & Mass of the lower leg ${ }^{1}$ & $\mathrm{~kg}$ & $\begin{array}{l}\text { Fixed }(0.061 \cdot \text { body } \\
\text { mass })^{4}\end{array}$ \\
\hline$p_{11}$ & $\begin{array}{l}\text { Position of the center of mass of } \\
\text { the lower leg }{ }^{1,3}\end{array}$ & $\mathrm{~m}$ & $\begin{array}{l}\text { Fixed }(0.525 \cdot \text { lower leg } \\
\text { length })^{4}\end{array}$ \\
\hline$r_{11}$ & $\begin{array}{l}\text { Radius of gyration of the lower } \\
\operatorname{leg}^{1}\end{array}$ & $\mathrm{~m}$ & $\begin{array}{l}\text { Fixed }(0.365 \cdot \text { lower leg } \\
\text { length })^{4}\end{array}$ \\
\hline
\end{tabular}

${ }^{1}$ Parameters for the lower leg included the shank and foot.

${ }^{2}$ With respect to hip joint.

${ }^{3}$ With respect to knee joint.

${ }^{4}$ Parameters according to the list compiled by Stein et al. [39] (see Table II). For some parameters they reported a range and in those cases we used the mean.

scale the literature-based estimates of the lower and upper leg mass and to get a better estimate of the subject's segment masses. We decided to scale the upper and lower leg equally (assuming that subjects with an above-average lower leg mass also exhibit an equally enlarged upper leg). The final estimate of a subject's upper and lower leg mass is thus obtained by multiplying the initial estimates from the literature with the scaling factor. The scaling factor was estimated based on the relax conditions (see the next paragraph).

For each subject, a total of six parameters $\left(K_{h}, B_{h}, K_{k}\right.$, $B_{k}, K_{c}$, and $B_{c}$ ) had to be estimated for each of the six conditions and one parameter ( $c$ : the scaling factor for $m_{\mathrm{ul}}$ and $m_{\mathrm{ll}}$ ) across conditions. The most reliable estimates for the segment mass and inertia are obtained at rest, when the contribution of joint stiffness and damping is small [31]. Therefore, we first performed the optimization for the three relax tasks. The optimized scaling factor was averaged over the three relax conditions and was used as a fixed parameter in the subsequent optimization of all six conditions.

Note that the identified stiffness parameters do not include the contribution of the gravitational stiffness. The gravitational stiffness is estimated based on the mass of the upper and lower leg, the scaling factor (see previous paragraph), and the joint angles [see (11), supplementary material].

To assess the sensitivity of the estimated parameters to the variation in response between the different cycles of the perturbation, the six parameters were also estimated per cycle. This resulted in six sets of the six parameters (per subject and per condition). Next, the standard deviation was calculated for every parameter (per subject and per condition). 
6) Model Validation: To obtain a measure of the accuracy of the model prediction we calculated the "goodness of fit" (GOF) in the frequency domain, where $100 \%$ reflects a perfect model fit. The GOF is defined according to:

$$
\mathrm{GOF}=\left(1-\frac{\sum_{f}\left|\log \left(\widehat{H}_{\text {human }}(f)\right)-\log \left(H_{\text {model }}(f)\right)\right|^{2}}{\sum_{f}\left|\log \left(\widehat{H}_{\text {human }}(f)\right)\right|^{2}}\right) \cdot 100 .
$$

The GOF was calculated for all 4 FRFs that comprise $\hat{H}_{\text {human }}$ separately. Since we used a logarithmic criterion function, we also calculated a logarithmic-based GOF.

7) Perturbation-Evoked Reflex Mechanisms: In our model, we do not attempt to decompose the stiffness into its reflexive and nonreflexive component. To determine the possible contribution of reflexive behavior to the joint stiffness, we calculated the NSR (in the frequency domain) of the EMG signal at the perturbed frequencies. This provides a measure of the inconsistency of the muscle activation levels at the perturbed frequencies. An inconsistent EMG response would suggest a small reflexive muscular contribution. The raw EMG recordings were bandpass filtered $(30-400 \mathrm{~Hz})$ with a second-order zero-lag Butterworth filter, full-wave rectified, and synchronized with the LOPES data. The lower bound of the filter was set at $30 \mathrm{~Hz}$ to ensure that all movement artifacts due to the perturbation (which has frequencies up to $10 \mathrm{~Hz}$ ) are removed. Since this is done before rectifying the EMG signal, this does not remove the actual muscle activation at the lower frequencies. The EMG data from the six perturbation cycles were Fourier transformed and the NSR was calculated for each muscle [in a way similar to that described in (4)].

\section{F. Statistics}

Stiffness and damping parameters were estimated in different leg postures. To assess the effect of task and leg orientation on the estimated stiffness and damping parameters, we performed a linear mixed model analysis with task (relax and position) and orientation ("end swing," "initial swing," and "mid swing") as fixed factors for each dependent variable. To account for the correlation between the repeated measurements within a subject, different intercepts were assumed for each subject by including the factor "subject" as a random factor into the analysis. If a main effect was found, post-hoc pairwise comparisons were used to locate the effect. Alpha was set at 0.05 and a Bonferroni correction was used to correct for multiple corrections. For the statistical analysis we used IBM SPSS statistics, version 22.0.

\section{RESULTS}

\section{A. Relax Task}

The angular response of the hip and knee joint to the six cycles of the perturbation signal was very consistent when subjects were instructed to relax (see Fig. 3 for a representative example). The consistent response of the subjects to the perturbation was also reflected in a low standard deviation and low NSR (see Fig. 3). It also shows that the noise levels are low and that there
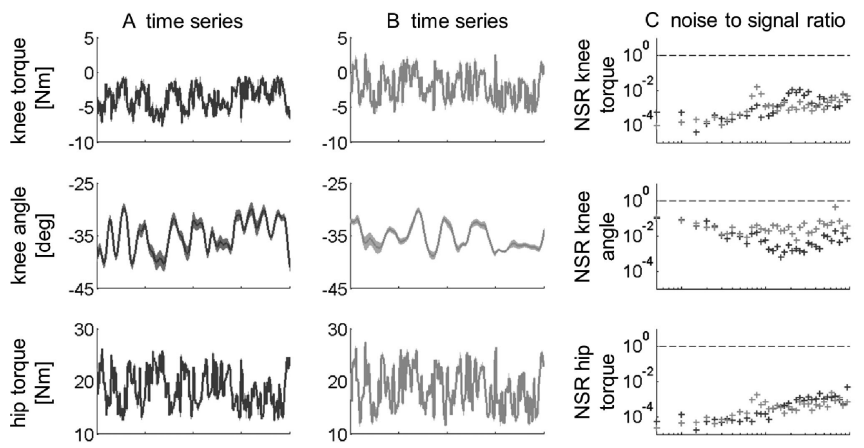

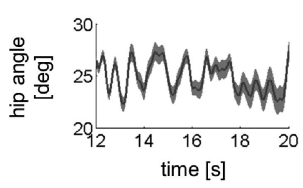

(a)

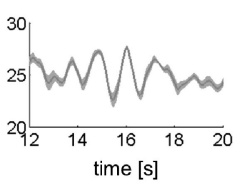

(b)

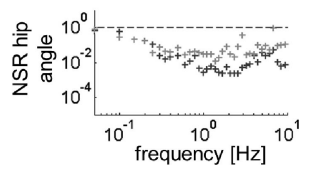

(c) perturbation round $2^{\text {nd }}$ perturbation round

Fig. 3. Time series and NSR during a relax task. An 8-s time series and NSR of one representative participant during a relax task (subject 5, leg orientation: "mid swing"). (a) Time series for the first perturbation. (b) Time series for the second perturbation. From top to bottom: knee torque perturbation, knee angular response, hip torque perturbation, and hip angular response. For the time series the mean is depicted by the solid line and the standard deviation over the 6 cycles by the shaded area. (c) NSR of the different signals, the dashed line depicts NSR $=1$.

is little time-variant behavior. Generally, the NSR of the angular response remained well below one and increased at the higher and lower frequency range (see Fig. 3). The NSR of the applied torque remained well below 1 at the full frequency range, indicating that the applied torque is very consistent over the cycles. Note that during the first perturbation, the hip and knee torque have opposite signs. Similar results were observed during the second perturbation (with a knee perturbation signal with equal sign, Fig. 3) and during the perturbations with the leg in the other configurations. The average NSR levels (averaged over all frequencies, perturbation cycles, subjects and leg configurations) were 0.21 for the hip angle and 0.10 for the knee angle. The similarity in the response to the different cycles of the perturbation signal was also confirmed by the low standard deviation over the six cycles (see Fig. 3(a, b) and Table II). For the relax tasks, the average peak-to-peak torque amplitudes (averaged over all perturbation cycles, subjects and leg configurations) was $13.4 \mathrm{Nm}$ for the hip and $6.3 \mathrm{Nm}$ for the knee, resulting in peak-to-peak angular displacement of $8.9^{\circ}$ and $15.0^{\circ}$ (see Table II). During the relax tasks, the average EMG levels were $3 \%$ of the MVC (see Fig. 4). We excluded two subjects from the analysis of the relax tasks, as they were not able to properly relax (EMG levels $>10 \% \mathrm{MVC}$ ).

\section{B. Position Task}

Generally, during the position task, the variability in response to the six cycles of the perturbation signal was larger than during the relax task (see Fig. 5 for a representative example). This is also reflected in higher NSR levels and higher standard deviations over the cycles, especially for the knee joint (see 
TABLE II

EXPERIMENTAL PARAMETERS

\begin{tabular}{|c|c|c|c|c|}
\hline & \multicolumn{2}{|c|}{ Relax task } & \multicolumn{2}{|c|}{ Position task } \\
\hline & Hip & Knee & Hip & Knee \\
\hline $\begin{array}{l}\text { Peak-to-peak angular } \\
\text { displacement [deg] }\end{array}$ & $8.9 \pm 1.5$ & $15.0 \pm 2.9$ & $9.2 \pm 1.0$ & $13.5 \pm 2.0$ \\
\hline $\begin{array}{l}\text { Peak-to-peak angular torque } \\
{[\mathrm{Nm}]}\end{array}$ & $13.4 \pm 1.2$ & $6.3 \pm 1.0$ & $19.9 \pm 0.2$ & $10.6 \pm 0.9$ \\
\hline NSR & $0.21 \pm 0.23$ & $0.10 \pm 0.04$ & $0.21 \pm 0.10$ & $0.54 \pm 0.26$ \\
\hline STD [deg] & $0.6 \pm 0.1$ & $1.3 \pm 0.8$ & $1.9 \pm 0.5$ & $2.3 \pm 0.7$ \\
\hline GOF (with bi-articular effect) & \multicolumn{2}{|c|}{ Relax task } & \multicolumn{2}{|c|}{ Position task } \\
\hline$H_{\theta_{\text {hip }} \rightarrow \tau_{\text {hip }}}$ & \multicolumn{2}{|c|}{$99 \pm 2.0$} & \multicolumn{2}{|c|}{$99 \pm 1.0$} \\
\hline$H_{\theta_{\text {hip }} \rightarrow \tau_{\text {knee }}}$ & \multicolumn{2}{|c|}{$84 \pm 19$} & \multicolumn{2}{|c|}{$87 \pm 12$} \\
\hline$H_{\theta \text { knee }} \rightarrow \tau_{\text {hip }}$ & \multicolumn{2}{|c|}{$73 \pm 15$} & \multicolumn{2}{|c|}{$91 \pm 6.5$} \\
\hline$H_{\theta_{\mathrm{knee}} \rightarrow \tau \text { knee }}$ & \multicolumn{2}{|c|}{$82 \pm 31$} & \multicolumn{2}{|c|}{$94 \pm 6.7$} \\
\hline $\begin{array}{l}\text { GOF (without bi-articular } \\
\text { effect) }\end{array}$ & \multicolumn{2}{|c|}{ Relax task } & \multicolumn{2}{|c|}{ Position task } \\
\hline$H_{\theta_{\mathrm{hip}} \rightarrow \tau_{\mathrm{hip}}}$ & \multicolumn{2}{|c|}{$99 \pm 0.23$} & \multicolumn{2}{|c|}{$99 \pm 1.0$} \\
\hline$H_{\theta_{\mathrm{hip}} \rightarrow \tau_{\mathrm{knee}}}$ & \multicolumn{2}{|c|}{$74 \pm 22$} & \multicolumn{2}{|c|}{$61 \pm 16$} \\
\hline$H_{\theta_{\mathrm{knee}}} \rightarrow \tau_{\mathrm{hip}}$ & \multicolumn{2}{|c|}{$63 \pm 19$} & \multicolumn{2}{|c|}{$69 \pm 8.6$} \\
\hline$H_{\theta_{\mathrm{knee}}} \rightarrow \tau$ knee & \multicolumn{2}{|c|}{$82 \pm 31$} & \multicolumn{2}{|c|}{$94 \pm 6.7$} \\
\hline
\end{tabular}

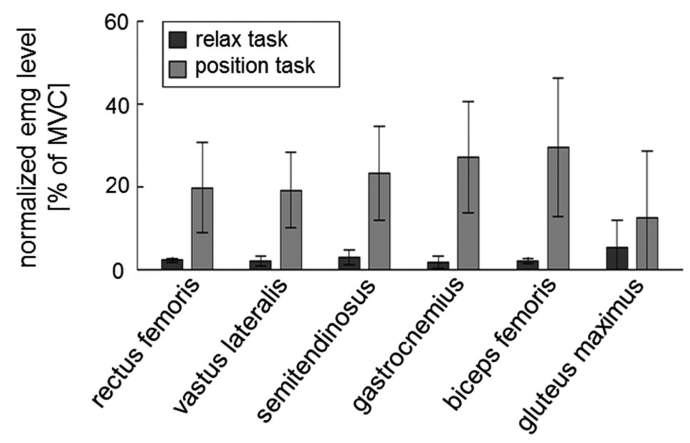

Fig. 4. EMG levels. Normalized EMG levels for the recorded muscles during the relax (dark gray) and position (light gray) tasks. The EMG levels are averaged over all perturbations, subjects, and leg configurations. The error bars indicate the standard deviation.

Table II). This is very likely caused by the fact that some subjects slowly start to deviate from the testing angle. With the help of the visual feedback subjects tried to compensate for this drift by small, low-frequency, angular corrections. These low-frequency angular corrections also explain the larger NSR at the lower frequencies (see Fig. 5). No consistent increase in the movement amplitude during the position task was found, which indicated that 1) fatigue was avoided and 2) a relatively constant co-contraction level was maintained. For the position tasks, the average peak-to-peak angular response was similar to the relax task, whereas a higher peak-to-peak torque perturbation was required to evoke the displacement (see Table II) due to the co-contraction. During the position tasks the average EMG levels were 22\% of the MVC levels (see Fig. 4). During the position task, where the subjects were asked to keep a constant level of co-contraction, they were able to keep their co-contraction level relatively constant. Although they did not receive any EMG feedback, the average variation (i.e., standard

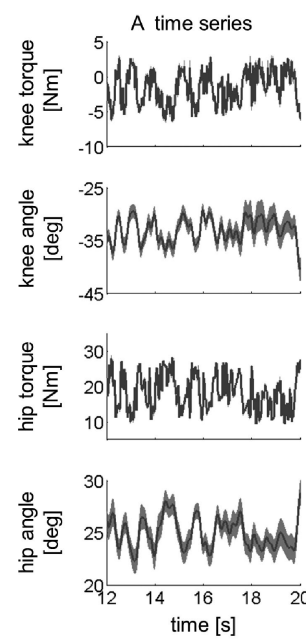

(a)
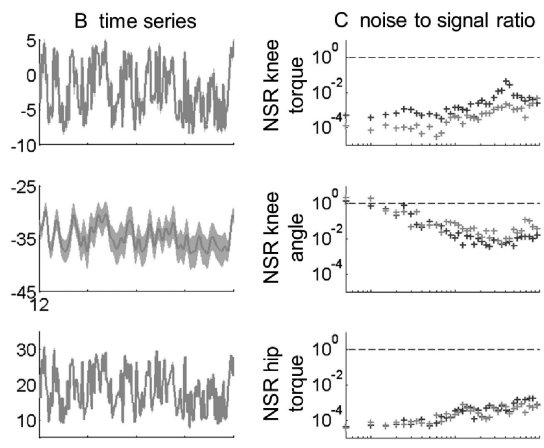

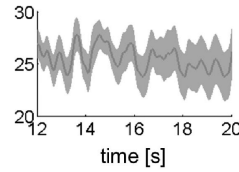

(b)

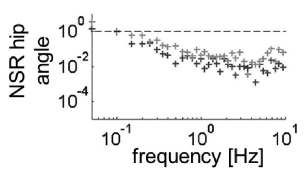

(c) $1^{\text {st }}$ perturbation round $2^{\text {nd }}$ perturbation round

Fig. 5. Time series and NSR during a position task. An 8-s time series and NSR of one representative participant during a position task (subject 5, leg orientation: "mid swing"). (a) Time series for the first perturbation. (b) Time series for the second perturbation. From top to bottom: knee torque perturbation, knee angular response, hip torque perturbation, and hip angular response. For the time series the mean is depicted by the solid line and the standard deviation over the six cycles by the shaded area. (c) NSR of the different signals. The dashed line depicts NSR $=1$.

deviation) of the mean EMG levels over the different cycles of the perturbation signal was only $4 \%$ of the MVC levels.

\section{Nonparametric FRFs}

From the two perturbations per condition, we calculated the FRF of the total system [human+LOPES see (2)] and subsequently the impedance FRF of the human leg (see the supplementary material). As expected, each of the four impedance FRFs resembles a second-order system (see Fig. 6 for a representative example). The phase, not shown here, starts at 0 and increases to $\pi$ at the higher frequencies. The relatively constant gain at the lower frequency range can be attributed to the stiffness, whereas the reduced gain in the mid-frequency range can be ascribed to the resonance point. The increasing gain with higher frequencies is caused by the leg's inertia. Generally, the task instruction had a clear effect on the estimated FRF. The position task resulted in higher gains for the lower and midfrequency range. The task instruction did not affect the high frequency part of the FRFs, which is dominated by the leg's inertia.

\section{Parametric Identification, Model Fit}

The estimated FRFs of the human leg could be described very well with the human leg model, for the relax as well as the position task (see Fig. 6 for a representative example). This is also reflected in a generally high GOF for each of the four TFs of the human leg model (see Table II). 

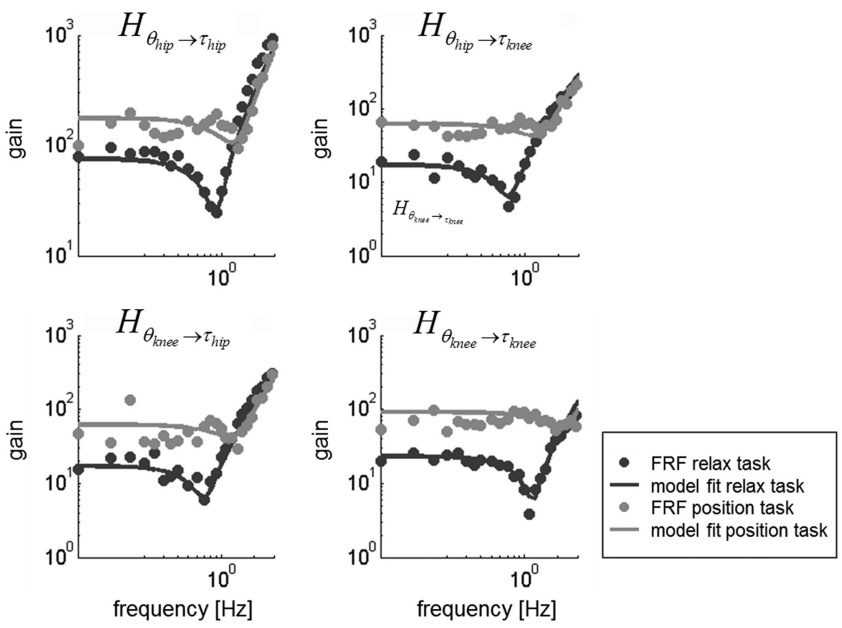

Fig. 6. Model fit during a relax and position task. Multiple-input-multipleoutput frequency response functions (FRFs) of one representative participant during a relax task (dark gray) and position task (light gray), (subject 6, leg orientation: "end swing"). $H_{\theta_{\mathrm{hip}} \rightarrow \tau_{\mathrm{knee}}}$ indicates the impedance FRF from hip angle to knee torque, etc. The solid lines represent the TF of the optimized human leg model. The FRFs and model fit are only shown for the frequency range that is used for the parameter optimization $(0.5-3 \mathrm{~Hz})$.
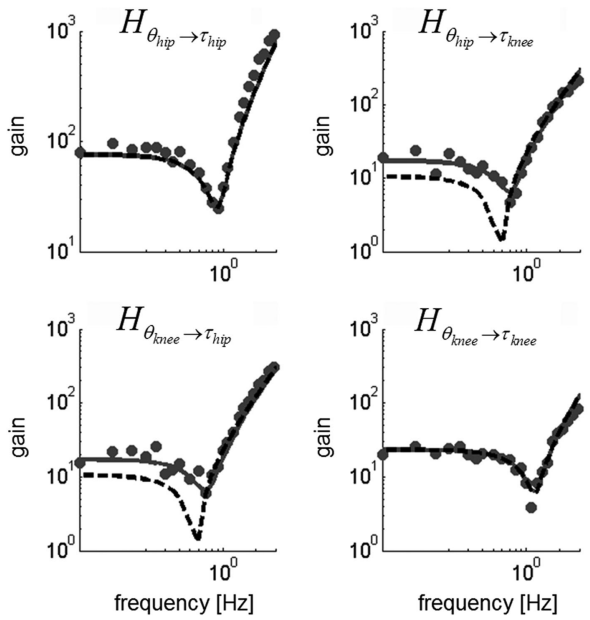

- FRF relax task

- model fit with

bi-articular effect model fit without bi-articular effect

frequency $[\mathrm{Hz}]$
TABLE III

RESULTS OF THE STATISTICAL ANALYSIS FOR EACH OF THE ESTIMATED PARAMETERS

\begin{tabular}{llll}
\hline \hline & Task & Orientation & Task $*$ Orientation \\
\hline$K_{h}$ & $F(1,30.6)=100.9$ & & $F(2,28.207)=3.491$ \\
& $P<0.0001$ & & $P=0.044$ \\
\hline$K_{c}$ & $F(1,32.8)=70.1$ & & \\
& $P<0.001$ & $P=0.041$ \\
\hline$K_{k}$ & $F(1,33.1)=110.0$ & & \\
& $P<0.001$ & & \\
\hline$B_{h}$ & $F(1,31.1)=141.0$ & & \\
& $P<0.001$ & & \\
\hline$B_{c}$ & $F(1,33.3)=95.6$ & $F(2,30.0)=9.3$ & \\
& $P<0.001$ & $P=0.001$ & \\
\hline$B_{k}$ & $F(1,33.3)=19.2$ & $F(2,30.1)=3.8$ & \\
& $P<0.001$ & $P=0.033$ & \\
\hline \hline
\end{tabular}

Values within parenthesis indicate the between-groups degrees of freedom and the within-groups degree of freedom.

initial parameters. Still, all optimizations resulted in the same set of estimated parameters. Generally, the scaling factor, which was used to scale the masses of the upper and lower leg, was below $1(0.87 \pm 0.10)$, indicating that the estimated masses are lower than reported in the literature [39].

Task instruction significantly affected all the stiffness and damping parameters. During the position task, all stiffness and damping parameters were significantly higher than during the relax task (see Table III).

There was no significant main effect of leg orientation for the estimated stiffness parameters, but there was a significant interaction effect for $K_{h}$ and $K_{c}$. Post-hoc analysis showed that both stiffness parameters were significantly higher in "end swing" orientation in comparison to "initial swing" orientation for the position task only $\left(K_{h}: \mathrm{P}=0.003, K_{c}: \mathrm{P}=0.004\right.$, see Fig. 8).

For the damping parameters, the orientation did have a significant effect for $B_{k}$ and $B_{c}$ (see Table III). The damping in "mid swing" was significantly higher than in "initial swing" ( $B_{k}: p=0.042, B_{c}, p<0.001$, see Fig 8).

To assess the sensitivity of the estimated parameters to the variation in response to the six different cycles of the perturbation, the six stiffness and damping parameters were also estimated per cycle. Fig. 8 shows the standard deviation of the stiffness and damping parameters (averaged over the subjects). In line with the NSR and STD parameters of the angular displacement (see Table II), there is more variation in the estimated parameters during the position task, compared to the relax task. Overall, for the stiffness parameter, the average standard deviation was around $15 \%$ of the estimated stiffness level. For the damping, the average standard deviation was around $20 \%$ of the estimated damping level. Generally, the NSR of the applied hip and knee torque is very low (see Figs. 3 and 5 for a representative example). Therefore, the difference in angular displacement over the cycles, and consequently the variation in the estimated stiffness and damping parameters, is most likely due to the variation in human response.
To avoid convergence of the fitting procedure to local minimum, we performed the optimization routine with different
Adding the bi-artcular stiffness and damping parameters, which reflects the effect of bi-articular muscles, clearly immodel fit. To illustrate this, we also show the bi-articular behavior is neglected, the 2 FRFs, $H_{\theta_{\text {hip }} \rightarrow \tau_{\text {knee }}}$ and $H_{\theta_{\mathrm{knee}} \rightarrow \tau_{\mathrm{hip}}}$, are not properly fitted by the TFs of the model (see Fig. 7 for a representative example during a relax task). This is also illustrated by a clear reduction of the GOF for both TFs when the bi-articular effect is neglected (see Table II).

\section{E. Parametric Identification, Estimated Model Parameters}




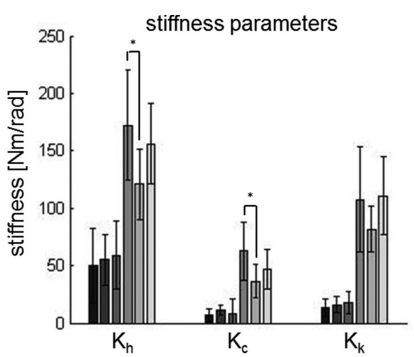

(a)

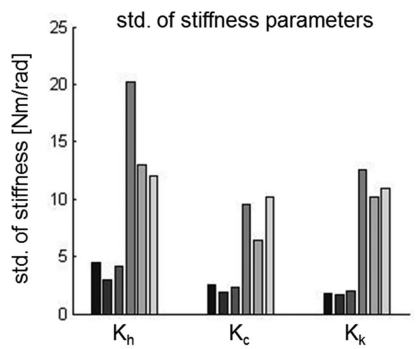

(c)

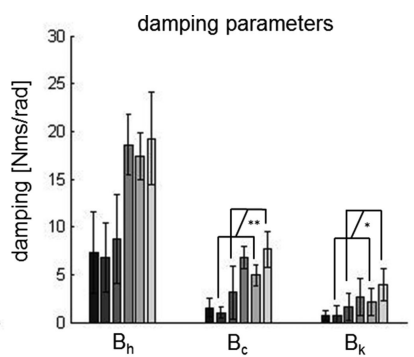

(b)

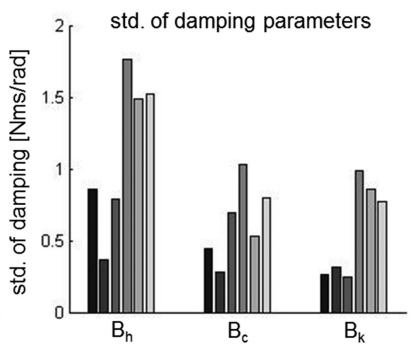

(d) task=relax, leg or.="end swing" task=relax, leg or.="initial swing" task=relax, leg or.="mid swing"

task=position, leg or.="end swing" task=position, leg or.="initial swing" $\square$ task=position, leg or.="mid swing"

Fig. 8. Stiffness and damping parameters. (a) Estimated stiffness and (b) damping parameters for the relax and position tasks in the different leg orientations. The error bars indicate the standard deviation over the subjects. For all parameters there was also a significant main effect of task instruction $(p<0.01)$. Other significant main effects and interaction effects are indicated with $*$ for $p<0.05$ and $* *$ for $<0.001$. (c) Standard deviation of the stiffness and (d) damping parameters estimated based on the different cycles of the perturbation signal (averaged over the subjects).

\section{F. Perturbation-Evoked Reflex Mechanisms}

The model presented in this study only considers intrinsic muscle stiffness, but some of this stiffness may have been caused by reflex pathways excited by the perturbation. During the relax tasks, for most muscles, the NSR was equally high for the perturbed frequencies and the nonperturbed frequencies. An inconsistent EMG response, and thus a high NSR, suggests a small reflexive muscular contribution. During the position task, there was a reduced NSR at the higher perturbed frequencies, compared to the nonperturbed frequencies (see Fig. 9 for a representative example). Although there seems to be a difference in EMG response during the relax and position task, the NSR is generally high, suggesting that the contribution of the reflex pathways to the stiffness is limited. In an effort to quantify this difference, we calculated the mean NSR over the 10 highest perturbed frequencies $(3.2-10 \mathrm{~Hz})$ and the nonperturbed frequencies. For the relax task, the mean NSR at the perturbed frequencies was $27.9 \pm 155.0$ (averaged over all muscles, subjects, and leg configurations) and $33.5 \pm 30.9$ at the nonperturbed frequencies. For the position task, the mean NSR at the perturbed frequencies was $6.07 \pm 19.2$ and $30.1 \pm 37.0$ at the nonperturbed frequencies.

\section{DISCUSSION AND CONCLUSION}

In this study, we present and evaluate a method to estimate hip and knee dynamic properties during multi-joint leg move-
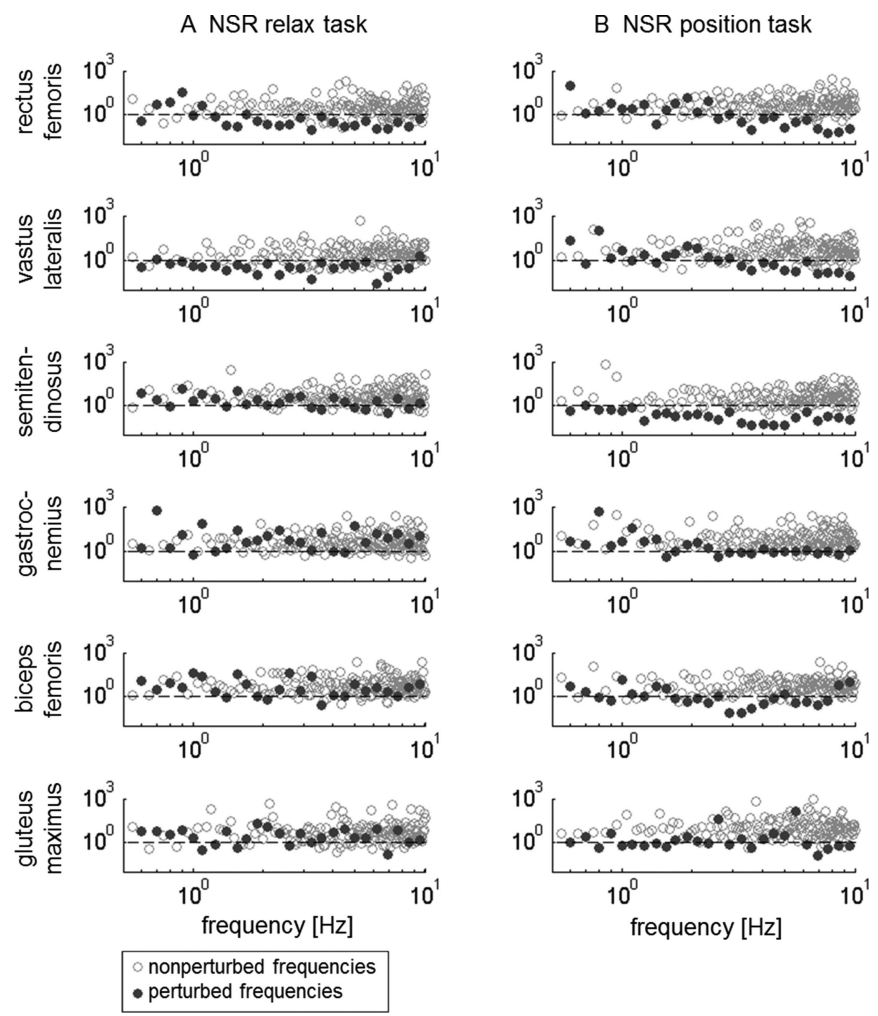

Fig. 9. NSR of the EMG activity. NSR of the EMG activity for the six recorded muscles of one representative participant (subject 5) during a relax task and during a position task (leg orientation: "mid swing"). The solid dark gray circles represent the NSR at the perturbed frequencies, and the dashed line depicts NSR $=1$.

ments by using a robotic gait trainer. The LOPES was used to apply continuous multisine torque perturbations to the hip and knee joint simultaneously. Frequency-domain MIMO linear system identification techniques were used to quantify the hip and knee dynamic (inter) joint stiffness, damping, and limb inertia. The results indicate that 1) all stiffness and damping parameters were significantly higher during the position task compared to the relax task, 2) the majority of the stiffness and damping parameters were not significantly affected by the leg orientation, and 3) including the effect of bi-articular muscles clearly improves the predictability of the multi-joint leg model. This section discusses these findings in further detail.

\section{A. Estimated Segment Masses}

In this study we scaled segment masses reported in the literature to fit the estimated FRFs. The scaling factor ranged between 0.75 and 0.94 , with an average of 0.87 , indicating that the estimated masses for most subjects were slightly lower than reported in the literature [39]. This may be related to the elderly subjects included, who may have relatively low upper- and lower-leg masses due to the reduction in muscle mass. Preliminary data (not shown) on nine young subjects (age 24.8 \pm 2.1 ), using the same protocol, resulted in an average scaling factor of $0.97 \pm 0.08$. 


\section{B. Effect of Task Instruction}

All stiffness and damping parameters that were used to model the multi-joint leg dynamics were significantly higher during the position task compared to the relax task. During the position task, subjects used co-contraction to reduce the displacement amplitude. Stiffness and damping are strongly related to muscle activation levels, due to a larger number of active cross-bridges. Overall, the stiffness and damping parameters increased $276 \%$ and $163 \%$ respectively, when the subjects were asked to minimize joint displacements.

1) Knee Stiffness: For the relax task, we found an average stiffness of $15.7 \mathrm{Nm} / \mathrm{rad}$, which seems reasonable taking into account the perturbation amplitude. It is known that estimates for joint stiffness decrease with perturbation amplitude, due to cross-bridge dynamics. Although these amplitude dependences are often associated with active muscles, they are also observed in passive isolated muscles [40]. More recently, amplitude dependences have also been reported for the human knee joint [5], [9]. In this study, we evoked angular displacements with a peak-to-peak amplitude of $15^{\circ}$. Studies that used small amplitude perturbations reported larger stiffness levels, up to around $75 \mathrm{Nm} / \mathrm{rad}$ [4], [5], [9]. Conversely, studies that applied pull or drop tests, and consequently evoked larger movements, reported slightly lower stiffness levels [39].

Only a few studies tried to measure knee stiffness during co-contraction. Pfeifer et al. [5] applied small amplitude position perturbations at different co-contraction levels. Their highest level of co-contraction corresponded to $10 \%$ MVC and resulted in a measured active stiffness ranging between 50 and $130 \mathrm{Nm} / \mathrm{rad}$. In our study, the estimated stiffness during the position task ranged between 54 and $203 \mathrm{Nm} / \mathrm{rad}$, but corresponded to higher co-contraction levels (22\% MVC). Note that this included also the passive stiffness, whereas the active stiffness reported by Pfeifer et al. is defined as the total stiffness minus the stiffness measured in the relax condition. Tai and Robinson [9] performed one of the few studies studying stiffness during a position task, as we did. They reported knee stiffness levels similar to ours, ranging between 130 (for low amplitude perturbations) and $60 \mathrm{Nm} / \mathrm{rad}$ (for large amplitude perturbations). Regretfully, they did not record the amount of muscle contraction. The fact that the abovementioned studies report similar stiffness levels, while using a wide variety of perturbation amplitudes also suggests that the amplitude-dependent change in knee stiffness primarily arises from passive properties of the joint. This has also been confirmed experimentally by Pfeifer et al. [5], who showed a drastic reduction in passive stiffness with increasing perturbation amplitude, whereas the active part of the total stiffness remained relatively constant. Similarly, Tai and Robinson [9] showed a reduction in total stiffness at larger amplitudes, which can predominantly be ascribed to a reduction of the passive stiffness.

2) Knee Damping: Knee damping has only been determined in a limited number of studies. The passive knee damping found in our study $(1.0 \mathrm{Nms} / \mathrm{rad})$ is in agreement with these studies. Tai and Robinson [41] and Zhang et al. [4], who used small amplitude position perturbations, reported passive damping pa- rameters of around 1 and 2, respectively. During the position task, we found an average damping of $2.9 \mathrm{Nms} / \mathrm{rad}$, which is 0.9 higher as reported by Tai and Robinson [41]. They also showed that the damping parameter is not affected by the perturbation amplitude (during passive conditions as well as co-contraction). This may explain why we found similar damping levels, even though we used perturbations with larger amplitudes.

3) Hip Stiffness and Damping: Hip dynamics were not studied as extensively as knee dynamics, and there are no known studies in which the hip joint was mechanically perturbed to estimate its stiffness and damping parameters. Torque-angle curves have been obtained while slowly moving the hip through its range of motion, but the stiffness based on the derivative of these curves is much lower than the stiffness during the relax task found in our study. As suggested in the introduction, this may be related to different mechanisms, which are active when the amplitude of the movement increases. In this study we found that the passive hip stiffness was much larger than the passive knee stiffness, possibly due to a larger amount of ligaments and muscle mass surrounding the hip joint.

\section{Effect of Leg Orientation on Knee Stiffness}

In this study we did not find a significant effect of leg orientation on passive knee stiffness. For the knee stiffness, during a relax task, Zhang et al. [4] and Tai and Robinson [9] report that the highest stiffness levels are recorded in the most extended positions $\left(<10^{\circ}\right.$ flexion). The reason that we did not find such a dependency is most likely due to the relatively high flexion angles that we tested $\left(15^{\circ}, 35^{\circ}\right.$, and $\left.55^{\circ}\right)$. Note also that, in order to make a fair comparison between passive knee stiffness levels, the hip angle should be taken into account, as this angle affects the pretension of bi-articular muscles and consequently the knee stiffness [29].

For the knee stiffness during a position task, Tai and Robinson [9] found that the stiffness was largest for the most extended positions, even after subtraction of the passive part. Although we did not find a significant effect of leg orientation on active knee stiffness, the largest average knee stiffness (after subtraction of the passive part) was found in the most extended position. The relatively large moment arm of the knee flexor muscles at $15^{\circ}$ of flexion [14] might explain the increase in stiffness in the extended position.

\section{Estimating Interjoint Stiffness and Damping}

All previous studies that assessed joint stiffness and damping in the lower extremities focused on a single joint. In this study, we perturbed the hip and knee joint simultaneously to assess multi-joint leg impedance. To model the recorded multi-joint leg impedance, we included a visco-elastic coupling between both joints. This model simulated the combined effect of biarticular muscles such as the hamstrings and the rectus femoris. The results show that these muscles create a substantial coupling between both joints, as reflected in the $K_{c}$ and $B_{c}$ parameters.

Hogan [42] showed that, for arm movements, the presence of bi-articular muscles around the shoulder and elbow dramatically increases the ability of the central nervous system to 
modulate the so-called endpoint stiffness (stiffness of the hand in the Cartesian space), through coordinated muscle activation of mono- and bi-articular muscles. He showed that without coupling between the joints it is not possible to achieve an isotropic endpoint stiffness (meaning that an input displacement in any direction would produce a proportional restoring force in exactly the opposite direction). In other words, without bi-articular muscles, the endpoint stiffness cannot be modulated in all directions, whereas humans have been shown to be capable of modulating their endpoint stiffness such that it specifically increases in the direction of the instability in the environment [43].

A similar mechanism might also be present in the lower extremities, to modulate the stiffness of the total leg. Simple bipedal spring-damper-mass models have already demostrated that the basic dynamics of walking and running can be explained by modeling the leg as one compliant element [44], [45], whose properties can be adjusted to cope with different environmental conditions or disturbances [46]. As with the upper extremities, the presence of a bi-articular coupling between the different leg joints increases the possibilities to modulate the compliance of the total leg. In fact, biped robots that are fitted with springs that correspond to the bi-articular muscles have been shown to improve the self-stabilizing characteristics for both walking and running gaits, compared to a setup in which only mono-articular springs are used [47]. Thus, besides the ability to transfer energy between different joints [48], which is often considered as their main task, bi-articular muscles might also contribute to a more stable gait.

\section{E. Limitations}

In this study, we performed perturbations during relaxed and co-contracted states. The co-contracted state was used to determine the maximum joint impedance. Still, this resulted in relatively low co-contraction levels (22\% MVC). This is probably related to the duration of the experiments. Subjects had to maintain a constant level of co-contraction throughout the trials. Since they are aware of the 1-min duration of the position tasks they may have chosen a relatively low co-contraction level. Still, the contraction levels are much higher than during the relax tasks (3\% MVC) and have a clear effect on the estimated stiffness. We also observed quite some variability in the EMG levels between subjects during the position tasks. The large intersubject variability in the active stiffness (see Fig. 8) could be a reflection of the different co-contraction levels. During the passive trials the intersubject variability in the estimated parameters was smaller.

Furthermore, the results do not allow conclusions about the repeatability of the measure, since the measurements were only performed in each subject once. Also, subsequent testing on neurological patients has to confirm its validity for measuring spasticity or abnormal muscle tone.

In this study we used the same scaling factor for the mass of the upper and lower leg. This scaling factor can also be considered as a limitation of this study, especially since the mass repartition can be different from one subject to another. This might be especially true for elderly people and for neurological patients.
In our study, we did not model reflexes that may have been evoked by the perturbation. Several studies showed that reflex activation contributes to joint stiffness for the ankle [49], [50]. For the knee joint, Pfeifer et al. [5] reported that there was no substantial relationship between perturbation and EMG during the passive trials, suggesting little reflex contribution, whereas during the active trials it was possible to predict $15-30 \%$ of the EMG variance. In our study, we did observe a reduced NSR of the EMG at the perturbed frequencies compared to the nonperturbed frequencies during the position task (see Fig. 9). However, the NSR was still high, suggesting that the reflexive contribution to the stiffness is limited. Reflex responses have been shown to decrease with amplitude [18]. Since we used perturbations which evoked relatively large movement amplitudes, and the contraction levels were relatively low, we did not expect a large reflexive contribution to the measured knee stiffness. Additionally, these reflexes have a decreased effect during sustained voluntary contractions [51] and have greater contributions for lower frequency bandwidth perturbations [52], whereas we also included higher frequencies.

Another limitation is that we could not include the ankle joint in the estimation of the multi-joint leg impedance. This was due to the fact that the current prototype of the LOPES does not have an actuated ankle joint. Measuring ankle stiffness would be of special interest since it is known that an abnormal ankle joint stiffness, due to neuromuscular disorders like SCI, stroke, cerebral palsy, or Parkinson's disease [22], [23], can affect walking ability. Still, the proposed method can be extended to include the ankle joint when future generations of the LOPES, or other gait trainers, are developed that do have ankle actuation.

\section{F. Future Directions}

This study shows that the LOPES can be used to estimate multi-joint impedance, as long as the equilibrium position of the joint, and the level of co-contraction, remains constant. The presented approach can be used in any gait trainer that is torque/force controlled, as long as the bandwidth of the applied torque/force exceeds the maximum frequency range that is required to appropriately excite the system and identify its dynamics. Measuring joint impedance with the same device that is used for robotic gait training enables convenient testing of joint properties as part of robotic gait training protocols. However, it is unknown, so far, whether the identified abnormal stiffness or damping measured under these conditions also hinders walking capacity. For example, increased stiffness is reported in neurological patients during relax conditions [22], [23], but little is known about its consequences (and even its occurrence) during gait. This information is critical in order to understand if, and how, their impairments affect walking ability.

In order to gain direct insight into how humans modulate their limb stiffness during different gait-related tasks, it would be of great benefit to estimate the joint stiffness during locomotion itself. However, direct measurement of the joint stiffness is complex and requires a device that is: 1) lightweight, 2) attaches rigidly to the limb so that accurate perturbations can be applied, and 3) is transparent when not applying the perturbations. Although the LOPES fulfills these requirements to a certain 
extent, joint impedance measurements during gait will probably start at a single joint level, requiring a more modular approach. Examples of such modular devices that can serve this purpose are the Bowden-cable-driven knee actuator used by Tucker et al. [53], or the pneumatically actuated AnkleBot presented by Lee et al. [54]. At our department, we are now developing a modular device, specifically dedicated to apply perturbations at the hip and knee, which can be used to estimate joint properties.

During walking, the time to apply stochastic perturbations is limited. Also, the joint impedance varies continuously due to: 1) variations in activation levels throughout the different gait phases and 2) a constant change in joint angle. Consequently, different system identification techniques will be required, such as the ones used by Ludvig et al. [55], [56], which allow the estimation of time-varying joint stiffness. We intend to further develop such methods and combine them with our method to estimate multi-joint impedance. Small-scale perturbations are preferably used, that minimally interfere with normal walking.

Such experiments will allow us to study to what extent neurological patients also show increased joint impedance during gait, and how it affects their walking ability. It may even be used to create patient-specific leg models and optimize the amount of applied torque by rehabilitation devices like the LOPES. Finally, such knowledge might prove to be beneficial for the development of intelligent, and energy efficient, prosthetic and orthotic devices.

\section{ACKNOWLEDGMENT}

The authors would like to thank J. Haarman for her assistance in collecting the data.

\section{REFERENCES}

[1] D. T. Westwick and E. J. Perreault, "Estimates of acausal joint impedance models," IEEE Trans. Biomed. Eng., vol. 59, no. 10, pp. 2913-2921, Oct. 2012.

[2] R. E. Kearney and I. W. Hunter, "System identification of human joint dynamics," Crit. Rev. Biomed. Eng., vol. 18, no. 1, pp. 55-87, 1990.

[3] P. L. Weiss, I. W. Hunter, and R. E. Kearney, "Human ankle joint stiffness over the full range of muscle activation levels," J. Biomech., vol. 21, no. 7, pp. 539-544, 1988 .

[4] L. Q. Zhang, G. Nuber, J. Butler, M. Bowen, and W. Z. Rymer, "In vivo human knee joint dynamic properties as functions of muscle contraction and joint position," J. Biomech., vol. 31, no. 1, pp. 71-76, 1998.

[5] S. Pfeifer, H. Vallery, M. Hardegger, R. Riener, and E. J. Perreault, "Model-based estimation of knee stiffness," IEEE Trans. Biomed. Eng., vol. 59, no. 9, pp. 2604-2612, Sep. 2012.

[6] G. L. Gottlieb and G. C. Agarwal, "Dependence of human ankle compliance on joint angle," J. Biomech., vol. 11, no. 4, pp. 177-181, 1978.

[7] P. L. Weiss, R. E. Kearney, and I. W. Hunter, "Position dependence of ankle joint dynamics-II. Active mechanics," J. Biomech., vol. 19, no. 9, pp. 737-751, 1986.

[8] R. E. Kearney and I. W. Hunter, "Dynamics of human ankle stiffness: Variation with displacement amplitude," J. Biomech., vol. 15, no. 10, pp. 753-756, 1982.

[9] C. Tai and C. J. Robinson, "Knee elasticity influenced by joint angle and perturbation intensity," IEEE Trans. Rehabil. Eng., vol. 7, no. 1, pp. 111-115, Mar. 1999.

[10] F. C. van der Helm, A. C. Schouten, E. de Vlugt, and G. G. Brouwn, "Identification of intrinsic and reflexive components of human arm dynamics during postural control," J. Neurosci. Methods, vol. 119, no. 1, pp. 1-14, 2002.
[11] W. Mugge, D. A. Abbink, and C. T. Helm, "Reduced power method How to evoke low-bandwidth behaviour while estimating full-bandwidth dynamics," in Proc. IEEE Int. Conf. Rehabil. Robot., 2007, pp. 575-581.

[12] P. L. Weiss, R. E. Kearney, and I. W. Hunter, "Position dependence of ankle joint dynamics-I. Passive mechanics," J. Biomech., vol. 19, no. 9 , pp. 727-135, 1986.

[13] P. M. H. Rack and D. R. Westbury, "The effect of length and stimulus rate on tension in the isometric cat soleus muscle," J. Physiol., vol. 204, pp. 443-460, 1969.

[14] M. D. Klein Horsman, H. F. J. M. Koopman, F. C. T. van der Helm, and H. E. J. Veeger, "The Twente lower extremity model: A comparison of muscle moment arms with the literature," Dissertation ch. 3, 2007.

[15] D. L. Morgan, "Separation of active and passive components of short-range stiffness of muscle," Amer. J. Physiol., vol. 232, no. 1, pp. C45-C49, 1977.

[16] P. M. Rack and D. R. Westbury, "The short range stiffness of active mammalian muscle and its effect on mechanical properties," J. Physiol., vol. 240, no. 2, pp. 331-350, 1974.

[17] E. de Vlugt, F. C. van der Helm, A. C. Schouten, and G. G. Brouwn, "Analysis of the reflexive feedback control loop during posture maintenance," Biol. Cybern., vol. 84, no. 2, pp. 133-141, 2001.

[18] A. Klomp et al., "Perturbation amplitude affects linearly estimated neuromechanical wrist joint properties," IEEE Trans. Biomed. Eng., vol. 61, no. 4, pp. 1005-1014, Apr. 2014.

[19] A. H. Hansen, D. S. Childress, S. C. Miff, S. A. Gard, and K. P. Mesplay, "The human ankle during walking: Implications for design of biomimetic ankle prostheses," J. Biomech., vol. 37, no. 10, pp. 1467-1474, 2004.

[20] E. J. Perreault et al., "Using estimates of dynamic endpoint stiffness to quantify the effects of triceps functional neuromuscular stimulation," in Proc. 2nd Annu. Conf. Int. Funct. Electr. Stimulation Soc., 2011, pp. 2-4.

[21] J. T. Blackburn, D. A. Padua, B. L. Riemann, and K. M. Guskiewicz, "The relationships between active extensibility, and passive and active stiffness of the knee flexors," J. Electromyography Kinesiol., vol. 14, no. 6 , pp. 683-691, 2004.

[22] M. M. Mirbagheri, H. Barbeau, M. Ladouceur, and R. E. Kearney, "Intrinsic and reflex stiffness in normal and spastic spinal cord injured subjects," Exp. Brain Res., vol. 141, no. 4, pp. 446-59, 2001.

[23] L. Galiana, J. Fung, and R. Kearney, "Identification of intrinsic and reflex ankle stiffness components in stroke patients," Exp. Brain. Res., vol. 165, no. 4, pp. 422-434, 2005.

[24] R. E. Kearney, P. L. Weiss, and R. Morier, "System identification of human ankle dynamics: Intersubject variability and intrasubject reliability," Clin. Biomech., vol. 5, no. 4, pp. 205-217, 1990.

[25] D. A. Abbink, "Task instruction: The largest influence on human operator motion control dynamics," in Proc. 2nd Joint EuroHaptics Conf. Symp. Haptic Interfaces Virtual Environ. Teleoperator Syst., 2007, pp. 206-211.

[26] P. A Forbes, R. Happee, F. C. T. van der Helm, and A. C. Schouten, "EMG feedback tasks reduce reflexive stiffness during force and position perturbations.," Exp. Brain Res., vol. 213, no. 1, pp. 49-61, 2011.

[27] R. J. H. Wilmink and T. R. Nichols, "Distribution of heterogenic reflexes among the quadriceps and triceps surae muscles of the cat hind limb," $J$. Neurophysiol., vol. 90, no. 4, pp. 2310-2324, 2003.

[28] A. Silder, B. Whittington, B. Heiderscheit, and D. G. Thelen, "Identification of passive elastic joint moment-angle relationships in the lower extremity," J. Biomech., vol. 40, no. 12, pp. 2628-2635, 2007.

[29] R. Riener and T. Edrich, "Identification of passive elastic joint moments in the lower extremities," J. Biomech., vol. 32, no. 5, pp. 539-544, 1999.

[30] B. Whittington, A. Silder, B. Heiderscheit, and D. G. Thelen, "The contribution of passive-elastic mechanisms to lower extremity joint kinetics during human walking," Gait Posture, vol. 27, no. 4, pp. 628-634, 2008.

[31] E. J. Perreault, R. F. Kirsch, and P. E. Crago, "Multi-joint dynamics and postural stability of the human arm," Exp. Brain Res., vol. 157, no. 4, pp. 507-517, 2004.

[32] E. De Vlugt, A. C. Schouten, and F. C. Van der Helm, "Closed-loop multivariable system identification for the characterization of the dynamic arm compliance using continuous force disturbances: A model study," $J$. Neurosci. Methods, vol. 122, no. 2, pp. 123-140, 2003.

[33] J. F. Veneman, R. Kruidhof, E. E. G. Hekman, R. Ekkelenkamp, E. H. F Van Asseldonk, and H. Van Kooij, "Design and Evaluation of the LOPES Exoskeleton Robot for Interactive Gait Rehabilitation," IEEE Trans Neural Syst Rehabil Eng, vol. 15, no. 3, pp. 379-386, 2007.

[34] H. J. Hermens et al., European Recommendations for Surface Electromyography, Results of the SENIAM Project. Roessingh Research and Development B.V, Enschede, The Netherlands, 1999. 
[35] E. H. F. van Asseldonk et al., "The effects on kinematics and muscle activity of walking in a robotic gait trainer during zero-force control," IEEE Trans. Neural Syst. Rehabil. Eng., vol. 16, no. 4, pp. 360-370, Aug. 2008.

[36] A. G. Jennings and B. B. Seedhom, "The measurement of muscle stiffness in anterior cruciate injuries-An experiment revisited," Clin. Biomech., vol. 13 , no. 2 , pp. $138-140,1998$

[37] R. Pintelon and J. Schoukens, System Identification: A Frequency Domain Approach. Piscataway, NJ, USA: IEEE Press, 2001

[38] T. A. Boonstra, A. C. Schouten, and H. van der Kooij, "Identification of the contribution of the ankle and hip joints to multi-segmental balance control," J. Neuroeng. Rehabil., vol. 10, no. 1, pp. 1-18, 2013.

[39] R. B. Stein et al., "Estimating mechanical parameters of leg segments in individuals with and without physical disabilities," IEEE Trans. Rehabil. Eng., vol. 4, no. 3, pp. 201-211, Sep. 1996.

[40] U. Proske and D. L. Morgan, "Do cross-bridges contribute to the tension during stretch of passive muscle?" J. Muscle Res. Cell Motility, vol. 20, nos. 5/6, pp. 433-42, 1999.

[41] C. Tai and C. J. Robinson, "Variation of human knee stiffness with angular perturbation intensity," in Proc. 17th Int. Conf. Eng. Med. Biol. Soc., 1995, pp. 1317-1318.

[42] N. Hogan, "The mechanics of multi-joint posture and movement control,' Biol. Cybern., vol. 331, pp. 315-331, 1985.

[43] D. W. Franklin et al., "Endpoint stiffness of the arm is directionally tuned to instability in the environment.," J. Neurosci., vol. 27, no. 29, pp. $7705-$ 7716, 2007.

[44] H. Geyer, A. Seyfarth, and R. Blickhan, "Compliant leg behaviour explains basic dynamics of walking and running," in Proc. Biol. Sci., vol. 273, no. 1603, pp. 2861-2867, 2006.

[45] E. Etenzi, S. Micera, and S. S. S. Anna, "Model of a stable passive bipedal walker provided with spring-damping legs," in Proc. Dyn. Walking Conf., 2007, pp. 5-6.

[46] M. Ernst, H. Geyer, and R. Blickhan, "Extension and customization of selfstability control in compliant legged systems," Bioinspiriration Biomimetics, vol. 7, no. 4, pp. 1-10, 2012.

[47] F. Iida, J. Rummel, and A. Seyfarth, "Bipedal walking and running with spring-like bi-articular muscles," J. Biomech., vol. 41, no. 3, pp. 656-667, 2008.

[48] J. V. A. N. Ingen, "From rotation to translation: Constraints on multijoint movements and the unique action," Human Movement Sci., vol. 8, pp. 301-337, 1989.

[49] T. Sinkjaer, E. Toft, S. Andreassen, and B. C. Hornemann, "Muscle stiffness in human ankle dorsiflexors: Intrinsic and reflex components," $J$ Neurophysiol., vol. 60, no. 3, pp. 1110-1121, 1988.

[50] M. M. Mirbagheri, H. Barbeau, and R. E. Kearney, "Intrinsic and reflex contributions to human ankle stiffness: Variation with activation level and position," Exp. Brain. Res., vol. 135, no. 4, pp. 423-436, 2000.

[51] G. Macefield, K. E. Hagbarth, R. Gorman, S. C. Gandevia, and D. Burke, "Decline in spindle support to alpha-motoneurones during sustained voluntary contractions," J. Physiol., vol. 440, pp. 497-512, 1991.

[52] F. C. T. van der Helm, A. C. Schouten, E. de Vlugt, and G. G. Brouwn, "Identification of intrinsic and reflexive components of human arm dynamics during postural control," J. Neurosci. Methods, vol. 119, no. 1, pp. 1-14, 2002.

[53] M. R. Tucker, A. Moser, O. Lambercy, J. Sulzer, and R. Gassert, "Design of a wearable perturbator for human knee impedance estimation during gait," in Proc. IEEE Int. Conf. Rehabil. Robot., 2013, pp. 1-6.

[54] H. Lee, S. Member, and N. Hogan, "Investigation of human ankle mechanical impedance during locomotion using a wearable ankle robot," in Proc. IEEE Int. Conf. Robot. Autom., 2013, pp. 2651-2656.
[55] D. Ludvig, T. S. Visser, H. Giesbrecht, and R. E. Kearney, "Identification of time-varying intrinsic and reflex joint stiffness," IEEE Trans. Biomed. Eng., vol. 58, no. 6, pp. 1715-1723, Jun. 2011.

[56] D. Ludvig, S. Pfeifer, X. Hu, and E. J. Perreault, "Time-varying system identification for understanding the control of human knee impedance,' in IFAC Proc., vol. 16, no. 1, pp. 1306-1310, 2012.

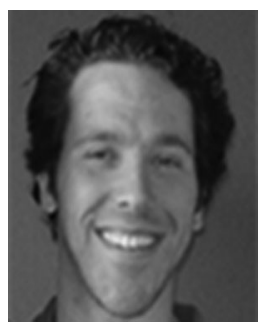

Bram Koopman was born in Twello, The Netherlands, on August 19, 1982. He received the M.Sc. degree in biomedical engineering and the $\mathrm{Ph} . \mathrm{D}$. degree from University of Twente, Enschede, The Netherlands, in 2008 and 2014, respectively.

$\mathrm{He}$ is a Project Engineer with Abbott Medical Optics, Groningen, The Netherlands.

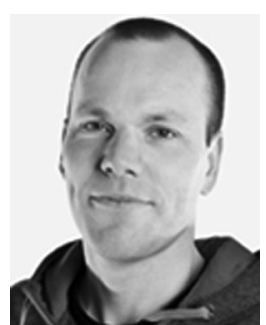

Edwin H. F. van Asseldonk (M'15) was born in Erp, The Netherlands, on March 28, 1978. He received the M.Sc. degree (honors) in human movement sciences from Free University, Amsterdam, The Netherlands, and the $\mathrm{Ph} \mathrm{D}$. degree in biomechanical engineering from University of Twente, Enschede, The Netherlands, in 2001 and 2008, respectively.

$\mathrm{He}$ is an Assistant Professor with University of Twente. His research interests include balance control, motor control in stroke patients, and rehabil itation robotics. He received the prestigious Dutch VENI grant in 2009

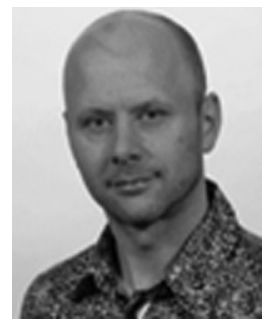

Herman van der Kooij (M'15) was born in 1970 He received the Ph.d. degree in biomechanics (Hons., cum laude) from the University of Twente in 2000.

$\mathrm{He}$ is a Professor in biomechatronics and rehabilitation technology with the Department of Biomechanical Engineering, University of Twente (UT), Enschede, The Netherlands, and with Delft University of Technology, Delft, The Netherlands. His research interests include the field of human motor control, adaptation, and learning; rehabilitation robots, diagnostic, and assistive robotics; virtual reality; rehabilitation medicine; and neurocomputational modeling. He has published more than 120 publications in the areas of biomechatronics and human motor control. He received the prestigious Dutch VIDI and VICI grants in 2001 and 2015, respectively.

Dr. Kooij is an Associate Editor of IEEE TRANSACTIONS ON BIOMEDICAL ENGINEERING and IEEE Robotics and Automation Letters, a Member of IEEE Engineering in Medicine and Biology Society Technical Committee of Biorobotics, and was a Member of several scientific program committees in the field of rehabilitation robotics, biorobotics, and assistive devices. At UT, he is the Founder and the Head of the Rehabilitation Robotics Laboratory that developed powered exoskeletons for the rehabilitation of upper and lower extremities. $\mathrm{He}$ is the Founder and the Head of the Virutal Reality Human performance laboratory that combines robotic devices, motion capturing, and virtual environments. 